\title{
ASSESSING THE IMPACT OF THE NUMBER OF GCPS ON THE ACCURACY OF PHOTOGRAMMETRIC MAPPING FROM UAV IMAGERY
}

\author{
Saponaro Mirko, Tarantino Eufemia, Reina Alessandro, \\ Furfaro Giuseppe, Fratino Umberto
}

Polytechnic University of Bari

\begin{abstract}
With recent advances in aerial data acquisition technologies from aircraft and Unmanned Aerial Vehicles (UAVs) very large datasets can be collected rapidly, covering significant surfaces with centimetre-scale resolution, with the consequence possibility to analyse geological structures of coastal areas within those datasets digitally. The monitoring of erosion mechanisms in fact requires high standards of precision to appreciate their effects. With the availability of a regular coastal monitoring programme being carried out in a large territory, UAVs can replace many of the conventional flights, with considerable advantages in the cost of data acquisition and without any loss in the quality of topographic and aerial imagery data.

Several works in literature have been focused on finding an effective and sustainable survey strategy to limit costs and work times. However, it is necessary to refine the photogrammetric mapping process to optimize its geometrical accuracy and ensure the multi-temporal and multi-scaling repeatability of final products.

The aim of this work is to test the accuracy obtainable from various photogrammetric workflows concerning the 3D modelling of a coastline area that is subject to hydrogeological instability. To this purpose, a set of image data acquired with a UAV, equipped with a non-metric camera and a low-accuracy GNSS/INS receiver, was processed. To maintain and test the accuracy of the whole process, an adequate number of Ground Control Points (GCPs) was acquired by means of a high precision GNSS surveying.
\end{abstract}

Keywords: GCPs, UAV imagery, PhotoScan, Direct-Georeferencing, Coastline monitoring

\section{Introduction}

The advent of UAV (Unmanned Aerial Vehicle) systems has fuelled a technological evolution of survey methodologies and data processing methods, extending as consequence the interconnections between different application fields (Green et al. 2019; Manfreda et al. 2018; Saponaro, Tarantino, and Fratino 2018). The possibility of also using low-cost and non-calibrated sensors has widened the geographic information range that can be acquired. Useful datasets can be obtained for photogrammetric 3D reconstructions or 2D topographic products as orthophotos and Digital Elevation Model (DEM), ensuring a geometric accuracy suitable for a GIS environment as well as for the cartographic field. In evidence, with recent advances in aerial data acquisition technologies from aircraft and UAVs, it is now possible to capture high-resolution rock surface images and analyse geological structures within those datasets digitally (Irvine, Roberts, and Oldham 2018). Coastal erosion management has become an increasingly crucial task in environmental protection and risk assessment (Papakonstantinou, Doukari, and Topouzelis 2017) as it requires multi-scale, suitable and up-to-date information on small movements. In the last few years, satellite images processing has proved to be a key technology in dealing with such problems but, as demonstrated in several works (Papakonstantinou, Topouzelis, and Pavlogeorgatos 2016), it has not been shown as an effective and sustainable solution in terms of spatial (over $50 \mathrm{~cm}$ ) and temporal resolution. A methodological advancement was achieved by adopting LiDAR technologies capable of generating models with subcentrimetric accuracy but downstream of a specialized field activity timely and costly expensive. Nowadays, very large UAV-datasets can be collected rapidly and inexpensively, covering significant surface areas with centimetre-scale resolution $(2-5 \mathrm{~cm})$ in a matter of minutes. Thus, georeferenced UAV photogrammetric products can be considered as practical to measure and depict the morphology of coastline stretches at risk, comparable with LiDAR works (Caroti, Piemonte, and Pieracci 2018; Talavera Madrigal et al. 2018), which allow the assessment of changes due to extreme wave phenomena or the securing from possible fall hazards of rock masses. 
However, upstream of the potentials highlighted, it is necessary to refine the photogrammetric mapping process to optimize its geometrical accuracy and ensure the multi-temporal and multi-scaling repeatability of final products. The accuracy of Structure from Motion (SfM) algorithm-derived products is highly variable, and the causes are still not fully understood (James et al. 2017). Different factors may affect the precision of UAV-derived data, such as flight parameters, image quality, processing software, the morphology of the studied area and, above all, the modalities of georeferencing. The ordinary use of Ground Control Points (GCPs) greatly improves the precision of SfM products, but at the same time their collection represents a laborious and time-intensive part of UAV campaigns (Manfreda et al. 2019). For this reason, several works in literature have been focused on finding an effective and sustainable GCPs-survey strategy to limit costs and work times (AgüeraVega, Carvajal-Ramírez, and Martínez-Carricondo 2017; Rangel, Gonçalves, and Pérez 2018; SanzAblanedo et al. 2018; Skarlatos et al. 2013). It has already been proved that at least three GCPs are necessary for the software platform to take advantage of such information, but the minimum number of GCPs needed to produce an optimal quality is still uncertain. On this topic, (Manfreda et al. 2019) proposes a useful table to review the accuracy values obtained by varying the GCPs number extrapolated by different UAV-works. (James et al. 2017) recommends a minimum of five GCPs, combined with an accurate calibration of the camera, for reliable achievements. In most cases, a significant accuracy improvement can be observed when passing from three to six GCPs, although results may obviously be influenced by the morphology of the study area, the equipment on-board and the processing workflow adopted. Therefore, developing a comprehensive understanding of the correlation between georeferencing and Bundle Adjustment (BA) processes of 3D models seems crucial.

In this work we tested the accuracy achievable from various photogrammetric workflows for a 3D coastline reconstruction, subject to hydrogeological instability. The monitoring of erosion mechanisms requires high standards of precision to appreciate their effects (Tarantino 2012; Valentini, Saponieri, and Damiani 2017; Valentini et al. 2017). To this purpose, image data acquired with a UAV, equipped with a non-metric camera and a low-accuracy GNSS/INS receiver, were processed using Agisoft PhotoScan software platform. To test the accuracy of the whole process, 20 GCPs were acquired by means of a high precision GNSS surveying. Several BA processes were run, varying the GCPs number used in the georeferencing step, thus recording the relative root-mean-square error (RMSE) values on the Control Points (CPs), considered as impact index on the quality of the final product.

\section{Methodology of research and materials}

The UAV surveyed area is a coastline stretch of about $400 \mathrm{~m}$, located South of Bari (Apulia, Italy) (Fig. 1). The territory in question is bounded towards the sea by a low coast with banks, with elevations between one 1 meter and 5 meters. This such morphological element is the latest in a series of slope falls associated with the Pleistocene marine abrasion terraces that characterize the coastal strip and which give the South-Eastern slopes of the Murge hills a their characteristic stepped profile.

The UAV flight was performed using a commercial quadcopter DJI Inspire 1 equipped with a nonmetric camera DJI ZenMuse X3 (focal length of $3.61 \mathrm{~mm}$, pixel size of $1.56 \mu \mathrm{m}$, effective pixels 12.4M). The flight was planned through the iOS app DJI Ground Station Pro, in order to maintain a nadir-position of the camera at a constant altitude of $70 \mathrm{~m}$ Above Ground Level (AGL), with an average Ground Sample Distance (GSD) equal to $0.03 \mathrm{~m} / \mathrm{pix}$. 89 waypoints were outlined in 7 strips, at a cruising speed of $5.5 \mathrm{~m} / \mathrm{s}$, scheduling an overlap of $80 \%$ between the images in the longitudinal direction of the flight and $70 \%$ in the lateral direction. Each image was related to the information about its synchronized positioning at the time of shooting. More precisely, the UAV used was equipped with a low-cost GNSS/INS receiver with an average accuracy of $2.54 \mathrm{~m}$ along the axes. This information is fundamental when processing imagery to obtain photogrammetric products for Direct Georeferencing (DG). 


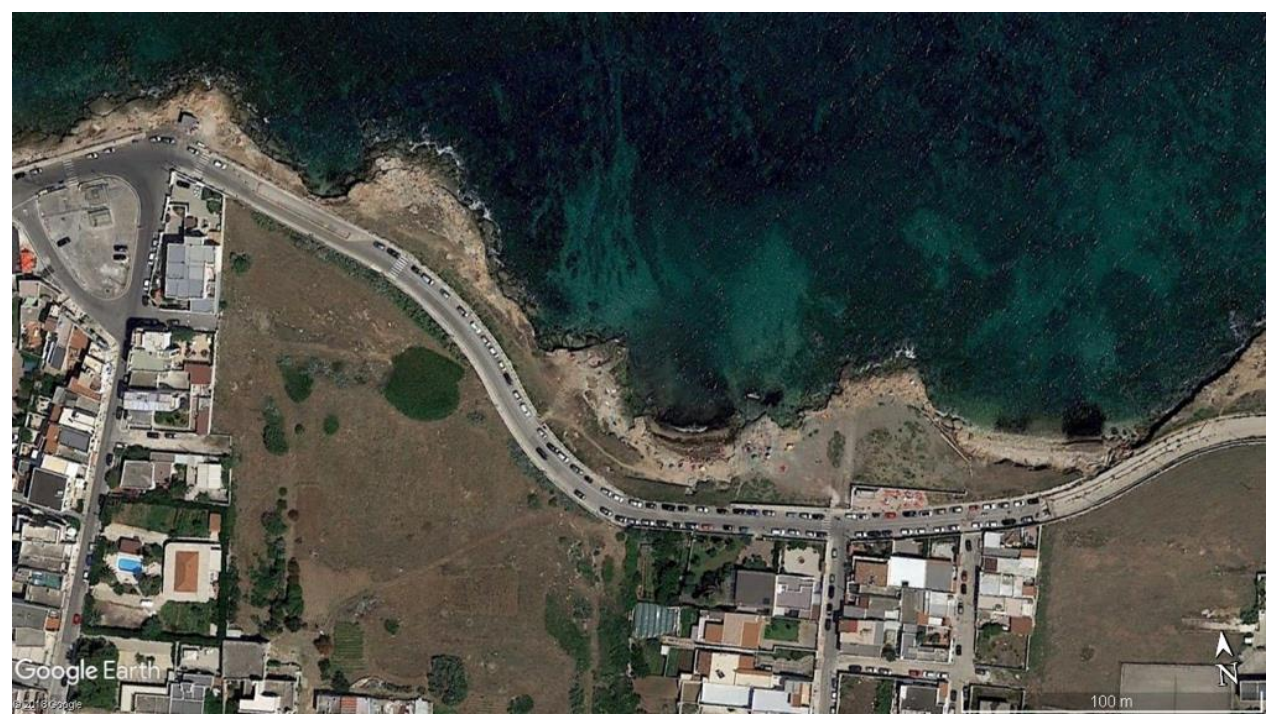

Fig. 1. Location of the study area (Google Earth image)

Two Chunks from the same imagery-dataset were generated through Agisoft Photoscan platform (v. 1.4.1). The first chunk was named "1- $70 \mathrm{~m}$ ", while the second one '2- $70 \mathrm{~m}$ TI", where TI means "treated images". In particular, each image was treated in order to mask water pixels and to assign an homogeneous brightness to the entire dataset (Fig. 2). As discussed in (Gonçalves and Henriques 2015), masking techniques may be used to avoid that large water bodies and other moving features being used in the point detection and matching step in order to achieve uncompromised results. Moreover, SfM algorithms can benefit from a regular brightness in the whole dataset, thus returning lower projection errors.

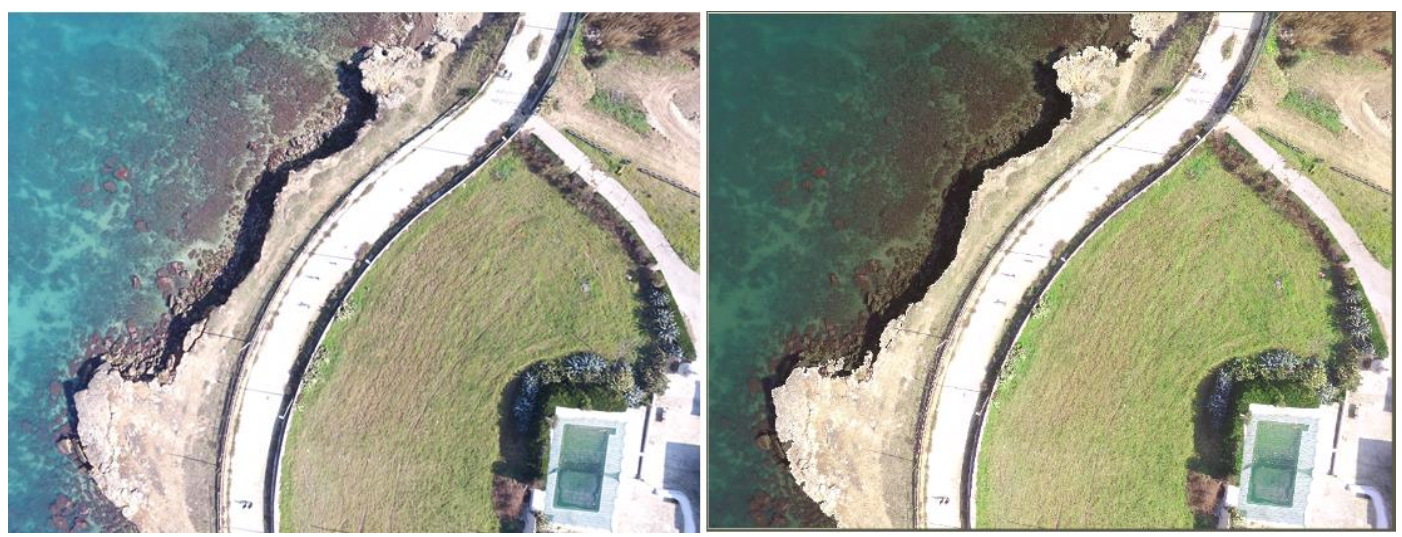

Fig. 2. Examples of masking techniques and exposure management of an image

A high-accuracy ground-truths measurement was performed: 20 GCPs were measured with Global Navigation Satellite System (GNSS) equipment, i.e. Leica Viva CS10/GS10 receivers. The points were detected with centimeter precisions $(0.02 \mathrm{~m})$ by performing measurements in Network Real Time Kinematic (nRTK) leaning to a local permanent station of the Leica SmartNet Italpos network, a commercial Countinously Operating Reference Station (CORS). The fixed points were searched in the area after mindful planning (visible edges, manholes, road signs, etc.), in order to have a uniform distribution along the coastline stretch under study. As suggested by (Scaioni et al. 2018), the transformation from ellipsoidal altitude obtained from GNSS survey to orthometric height is a key topic, because the latter is indispensable to compare the derived products. In this study case the ellipsoidal altitude was transformed into orthometric height using an accurate local geodetic model, comparing these heights with the barometric values acquired by the UAV. A systematic vertical shift was registered due to the its low-accuracy. The most diffused solution, in such case, is to acquire a 
UAV image on the ground before starting the mission or after the landing near or on a measured GCP in order to record the shift between the altitude coordinates. The value will then be fundamental to analyze the results of the DG.

Firstly, a reasonable setting of the system and the workspace was chosen (Mayer, Gomes Pereira, and Kersten 2018). To remove images that were not suitable for photogrammetric process, the mean image quality was computed using the PhotoScan tool that explores images one by one, estimating for each image a value between a low quality of 0 and and high quality equal to 1 . No images were removed, having obtained values higher than the threshold of 0.5 (mean value equal to 0.862), moreover no evident distortions or blur effects were present in the imagery.

In the Reference Settings pane, the workspace was then set: the reference system WGS 84 (EPSG::4326) was indicated for the imagery due to the geo-tags reference system, whereas the reference system RDN2008/UTM zone 33N (NE) (EPSG::6708) was set for the surveyed GCPs. Following the prompts in (Mayer, Gomes Pereira, and Kersten 2018), a value of $3 \mathrm{~m}$ was put in Accuracy Image $(\mathrm{m})$ parameter as a conservative setting, although the UAV-positioning accuracy value was known as equal to $2.54 \mathrm{~m}$. Furthermore, the precision of this value was related to the receiver rate of position measurements per second $(\mathrm{Hz})$ and hence to the mean speed of the vehicle. Although the imagery was acquired in a hovering 'stop\&go' mode, a conservative value was preferred as precautionary. To follow, in Accuracy Image (deg), the default value of $10 \mathrm{deg}$ was retained, since there was no manufacturing information, to also include the negative effects caused by the low accuracy of the IMU-onboard. A mean value of $0.02 \mathrm{~m}$ was fixed under the Precision Marker (m) option: as already mentioned, this parameter represents the mean precisionof GCPs coordinates in the object space. Finally, for the calibration of the image coordinates in the displayed workspace, a more reasonable Marker Precision (pix) value of 0.5 pix was selected, to define the effective ability of the operator to identify the target in the image. The accuracy of the tie points was retained at the default value of 1 pix. This parameter depends on the image quality since tie point positions are detected on the base of the key features showing in the images. Tie points were accurately localized when the images were sharp. A subpixel value avoids the distortion of the photogrammetric block, but at the same time it cuts-off the points re-projection number. The accuracy of Tie points, the Precision Marker (pix) and the Precision Marker (m) parameters are fundamental to generate the variancecovariance matrix within the stochastic model and therefore functional to the BA optimization.

Camera calibration, as mentioned in (James et al. 2017), is a critical step in order to obtain a welldefined interior orientation of imagery, and thus to avoid the error propagation in the final accuracy. There are various ways of calibrating the camera and each of them affects the final results of accuracy. It is often preferred to apply the parameters obtained by a self-calibration using the algorithms implemented in the software platform, noting that this produces optimized values compared to those obtainable from a raw calibration in the laboratory. Therefore, in the Camera Calibration panel, the task of estimating the aforementioned parameters was assigned to the software in the Camera Alignment process, which was then optimized in the BA procedures of the following phase.

Another basic operation was the set-up of the GPS/INS Offset in order to reach the lever-arm value corrections measured in the laboratory. In particular, the rough displacement vector $(\mathrm{X}, \mathrm{Y}, \mathrm{Z})$ measured was $(0.005,0.10,0.25) \mathrm{m}$ with a precision of about $0.01 \mathrm{~m}$, while the IMU values calibration (yaw, pitch, roll) was 0 deg with an accuracy considered equal to $2 \mathrm{deg}$. In the following phases these values were optimized too.

Once the workspace was structured, the Camera Alignment process was launched in 'Highest' accuracy, choosing the Limit of Key Points and Tie Points as equals to 0. Photoscan generated the sparse points clouds and, at same time, estimated the interior and exterior orientation, evaluating a systematic error compensation such as the lens non-linear distortions. The points clouds obtained showed a Gradual Selection as reported in Table 1. Briefly, the inaccurate points computed were cleaned out from the clouds for accuracy optimization and 20 GCPs were identified in the imagery, assigning a marker in the workspace displayed to the fixed points on the ground. 
Gradual Selection step: tie points resulting from the removing of inaccurate points for accuracy optimization

\begin{tabular}{|lcccc|}
\hline \multicolumn{1}{|c}{ Chunk } & $\begin{array}{c}\text { Initial } \mathbf{n}^{\circ} \\
\text { tie points }\end{array}$ & $\begin{array}{c}\text { Reconstruction } \\
\text { Uncertainity }\end{array}$ & $\begin{array}{c}\text { Projection } \\
\text { Accuracy }\end{array}$ & $\begin{array}{c}\text { Reprojection } \\
\text { Error }\end{array}$ \\
\hline $1-70 \mathrm{~m}$ & 1451042 & 1118218 & 999538 & 933305 \\
$2-70 \mathrm{~m} \mathrm{TI}$ & 1315819 & 1020920 & 932268 & 872548 \\
\hline
\end{tabular}

In order to comprehend the impact of the number of GCPs on the final accuracy achievable at the end of the georeferencing process, 21 copies were generated for each Chunk, in a total of 42 sparse points clouds in the workspace. These clouds were georeferenced by implementing from a maximum of 20 to a minimum of 0 GCPs, thus also analysing the cases of Complete Indirect Georeferencing (CIG) and DG respectively. The implemented GCPs were chosen in such a way to maintain a homogeneous distribuition between central and border zones (Fig. 3). The remaining number of the non-implemented fixed points in each process were consequently defined as Check Points (CPs).

The BA was launched through the Optimize Cameras process. During these step, the BA algorithms regulate estimated points coordinates, the values of camera parameters and lever-arm offset, reducing the sum of re-projection and reference coordinate misalignment errors.

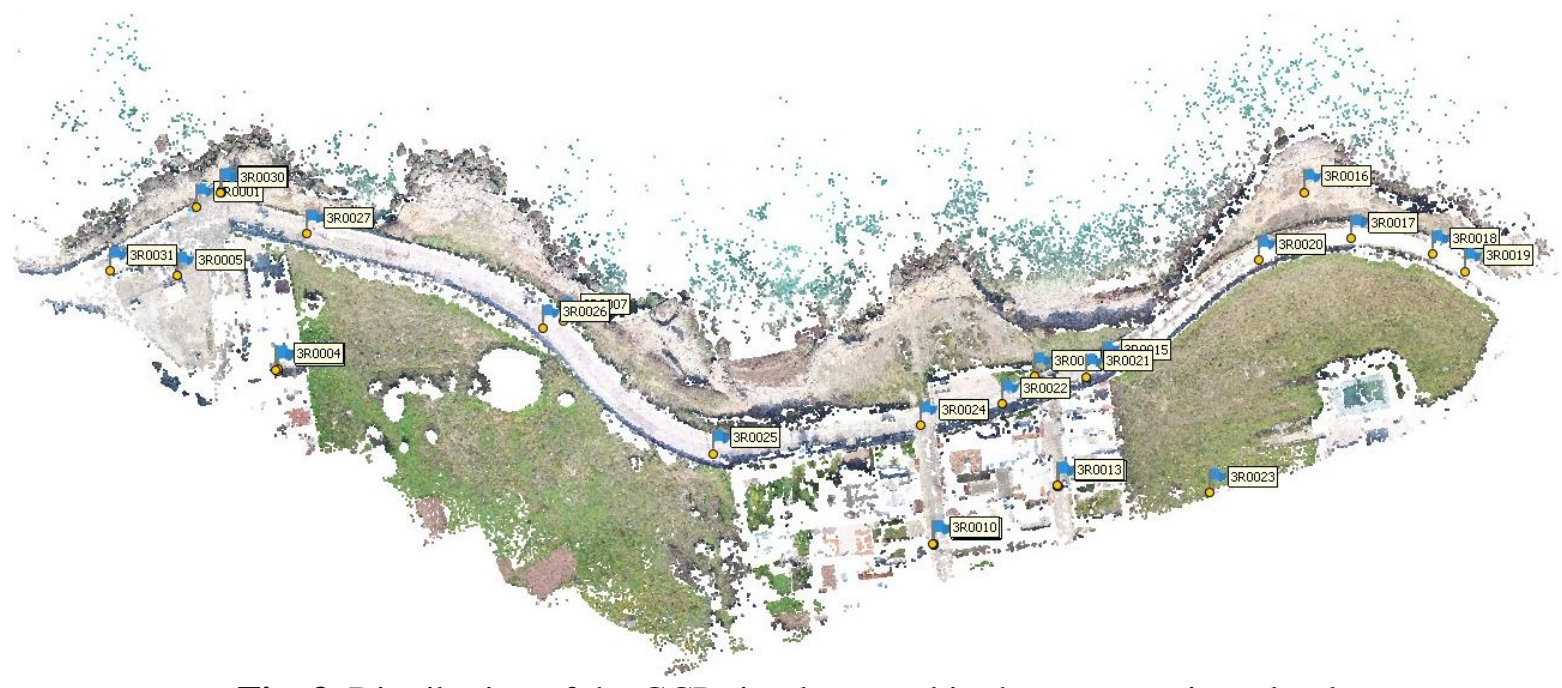

Fig. 3. Distribution of the GCPs implemented in the sparse points cloud

\section{Discussions and results}

Launching the alignment of cameras in each Chunk can have a positive impact when managing a cleaning clouds process utilizing three picking criteria implemented in the Gradual Selection tool.

The Reconstruction Uncertainty criterion allows to remove points with low base-to-height ratios. Namely, tie points situated in the margin areas of images generally have a higher degree of reconstruction uncertainty than central ones, due to the low sidelapping of the images. Removing such points doesn't impact on the accuracy, but it does lighten the clouds.

The second criterion applied, named Projection Accuracy, allows to recognise less reliable tie points. Setting this parameter as equal to 10 means that the points recognized have an uncertainty 10 times higher than the points characterized by a minimum uncertainty.

The Re-projection Error criterion was run to remove erroneous points with large residuals implying the largest direct influence on the root-mean-square-error (RMSE) of the GCPs and CPs. This parameter improved the orientation parameters conspicuously.

To follow, the impact of the number of GCPs on the geometric quality of the derived photogrammetric products was also studied. Several criteria discussed in many scientific works allow the assessment of the model quality in terms of its 3D coordinates accuracy (Manfreda et al. 2019). Commonly, these checks are based on comparing some estimated ground-points by SfM-algorithms and the same points measured with high accuracy, i.e. in a GNSS-campaign. Usually, the planimetric 
and altimetric accuracies are explained in terms of root-mean-square error (RMSE) registered on CPs or GCPs. The RMSE value can be considered as a cumulative result of all errors, i.e. both random and systematic errors with a Gaussian distribution (Saponaro, Tarantino, and Fratino 2018), and accordingly representative of the absolute accuracy of each point. Fig. 4 shows a complete view of mean errors, max e min errors registered in CPs for each process. The mean error represents the effective systematic displacement between the estimated points and the truth-points measured: a stable and recognizable discrepancy. A RMSE value much higher than the recorded mean error would indicate a prevalence of accidental errors and therefore a strong instability of the results. Vice versa, RMSE and mean errors comparable, verified by the bias values (min-max errors), clarify the statistical robustness of results.

Fig. 4 shows the $\mathrm{RMSE}_{\mathrm{xyz}}$ values in CPs related to the 20 processed Chunks respectively, escluding the CIG case: its results are shown in Table 2.

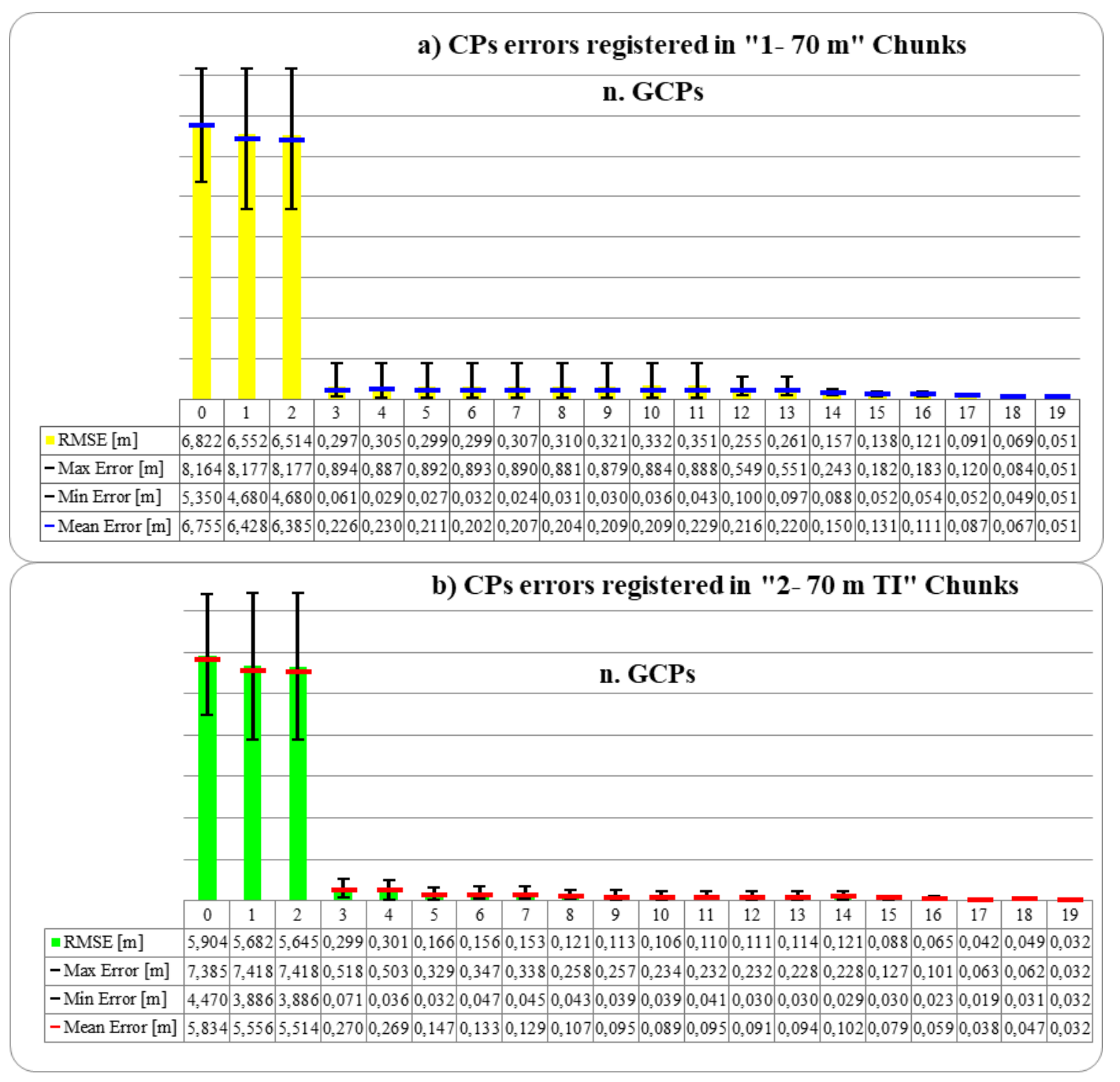

Fig. 4. a) CPs errors registered in "1- 70 m" Chunks varying the GCPs number implemented b) CPs errors registered in "2- $70 \mathrm{~m}$ TI" Chunks varying the GCPs number implemented

Analyzing the DG case, i.e. without GCPs assistance and only using only the image positional tags checked in the box pane before starting the process, the accuracies achieved show large gaps for a possible use in the cartographic field. The low accuracy of the sensors mounted does not allow the achievement of a high positional accuracy in the DG and, on the other hand, the lack of raw position data does not allow to identify the causes in order to improve its estimates. 
It is also necessary to consider an efficient amount of GCPs in the data georeferencing workflow to achieve highly accurate geospatial products.

Overall, the two datasets show a similar behavior in terms of number of GCPs implemented. There is a reduction in $\mathrm{RMSE}_{\mathrm{xyz}}$ values in the transition from a number of GCPs used from 2 to 3, with a RMSE $_{\text {xyz }}$ leap of about $5.5 \mathrm{~m}$. The following configurations attest the same order of magnitude achieved with slight centimetric fluctuations, linked to the Re-projection Errors in the images of GCPs implemented.

Table 2

Full description of the RMSE $E_{x y z}$ values [m] and the Re-projection Errors [pix] recorded in the 42 generated Chunks. The red box highlights the results considered as optimal for the model georeferencing. In the green box, the results obtained for the CIG cases.

\begin{tabular}{|c|c|c|c|c|c|c|c|c|c|c|c|}
\hline \multirow[b]{2}{*}{ n.GCPs } & & \multicolumn{2}{|c|}{ 1- $70 \mathrm{~m}$} & \multicolumn{2}{|c|}{$2-70 \mathrm{~m} \mathrm{TI}$} & & & & & & \\
\hline & & $\begin{array}{c}\operatorname{RMSE}_{\mathrm{xyz}} \\
\text { (m) }\end{array}$ & $\begin{array}{l}\text { Error } \\
\text { (pix) }\end{array}$ & $\begin{array}{c}\operatorname{RMSE}_{\mathrm{xyz}} \\
\text { (m) }\end{array}$ & $\begin{array}{l}\text { Error } \\
\text { (pix) }\end{array}$ & & & & & & \\
\hline \multirow{2}{*}{0} & GCPs & & & & & \multirow{2}{*}{11} & GCPs & 0.076 & 0.639 & 0.088 & 0.487 \\
\hline & $\mathrm{CPs}$ & 6.822 & 2.466 & 5.904 & 0.481 & & CPs & 0.351 & 3.917 & 0.110 & 0.484 \\
\hline \multirow{2}{*}{1} & GCPs & 6.820 & 0.390 & 5.908 & 0.484 & \multirow{2}{*}{12} & GCPs & 0.244 & 2.990 & 0.087 & 0.507 \\
\hline & $\mathrm{CPs}$ & 6.552 & 2.536 & 5.682 & 0.480 & & CPs & 0.255 & 0.502 & 0.111 & 0.445 \\
\hline \multirow{2}{*}{2} & GCPs & 7.015 & 0.312 & 6.106 & 0.431 & \multirow{2}{*}{13} & GCPs & 0.236 & 2.888 & 0.084 & 0.504 \\
\hline & CPs & 6.514 & 2.656 & 5.645 & 0.487 & & CPs & 0.261 & 0.493 & 0.114 & 0.442 \\
\hline \multirow{2}{*}{3} & GCPs & 0.096 & 0.409 & 0.099 & 0.414 & \multirow{2}{*}{14} & GCPs & 0.263 & 2.835 & 0.081 & 0.502 \\
\hline & CPs & 0.297 & 2.800 & 0.299 & 0.498 & & CPs & 0.157 & 0.495 & 0.121 & 0.441 \\
\hline \multirow{2}{*}{4} & GCPs & 0.085 & 0.415 & 0.088 & 0.501 & \multirow{2}{*}{15} & GCPs & 0.256 & 2.752 & 0.089 & 0.513 \\
\hline & CPs & 0.305 & 2.894 & 0.301 & 0.475 & & CPs & 0.138 & 0.395 & 0.088 & 0.380 \\
\hline \multirow{2}{*}{5} & GCPs & 0.077 & 0.415 & 0.084 & 0.493 & \multirow{2}{*}{16} & GCPs & 0.249 & 2.706 & 0.087 & 0.507 \\
\hline & CPs & 0.299 & 2.986 & 0.166 & 0.480 & & CPs & 0.121 & 0.391 & 0.065 & 0.395 \\
\hline \multirow{2}{*}{6} & GCPs & 0.072 & 0.435 & 0.078 & 0.487 & \multirow{2}{*}{17} & GCPs & 0.245 & 2.689 & 0.087 & 0.503 \\
\hline & CPs & 0.299 & 3.047 & 0.156 & 0.484 & & CPs & 0.091 & 0.365 & 0.042 & 0.399 \\
\hline \multirow{2}{*}{7} & GCPs & 0.071 & 0.688 & 0.078 & 0.497 & \multirow{2}{*}{18} & GCPs & 0.239 & 2.605 & 0.084 & 0.493 \\
\hline & CPs & 0.307 & 3.168 & 0.153 & 0.477 & & CPs & 0.069 & 0.369 & 0.049 & 0.432 \\
\hline \multirow{2}{*}{8} & GCPs & 0.088 & 0.681 & 0.102 & 0.519 & \multirow{2}{*}{19} & GCPs & 0.234 & 2.558 & 0.082 & 0.494 \\
\hline & CPs & 0.310 & 3.394 & 0.121 & 0.457 & & CPs & 0.051 & 0.422 & 0.032 & 0.392 \\
\hline \multirow{2}{*}{9} & GCPs & 0.084 & 0.668 & 0.099 & 0.508 & \multirow{2}{*}{20} & GCPs & 0.227 & 2.466 & 0.081 & 0.486 \\
\hline & CPs & 0.321 & 3.470 & 0.113 & 0.464 & & CPs & & & & \\
\hline \multirow{2}{*}{10} & GCPs & 0.080 & 0.662 & 0.093 & 0.506 & & & & & & \\
\hline & CPs & 0.332 & 3.572 & 0.106 & 0.465 & & & & & & \\
\hline
\end{tabular}

The optimal solutions were searched, based on the simultaneous occurrence of low RMSE values on both CPs and GCPs: a necessary condition to demonstrate the consistency of the RMSE values recorded on the CPs. The values in Table 2 show an optimal geometrical accuracy achieved with 6-7 GCPs implemented for both Chunks, thus confirming the results achieved by (James et al. 2017).

Focusing on the differences between the two processed Chunks, some considerations can be drawn. With reference to Table 2, Chunks "2 - 70 m TI" show Re-Projection Errors (pix), for both ground points, lower than the Chunks "1- $70 \mathrm{~m}$ " and, at the same time, these improve the geometric 
accuracy achievable on CPs. It can be therefore maintained that the techniques of masking and brightness adjustment effectively improve the detecting and matching of tie points, providing more robust results. On the other hand, however, these techniques may undermine the development of complete 3D models by not accurately masking particular areas. In the case of this work a punctual masking of the rocky coastline could only be achieved through a complicated and above all timeconsuming process. Its use is therefore recommended in morphologically regular areas.

\section{Conclusions and proposals}

A photogrammetric processing workflow was explored in this study for the accuracy optimization of UAV-based products, analysing a coastline stretch subject to hydrogeological instability.

Generally. the common low-precision equipment on board of a UAV does not guarantee the achievement of precision standards for topographic purposes: sparse points clouds have to be managed by firstly identifying and then removing tie points with low quality matching and high re-projection errors. A reasonable management of the clouds may impact on the model malleability and therefore on the final accuracy of results achievable. Further benefits can be observed in the final results as a result of masking and brightness adjustment techniques applied on the image dataset. These techniques, as demonstrated in this study, improve the final RMSE values by almost $50 \%$ but, at the same time, they may prove unsuitable in morphologically complicated scenarios. Their use is therefore recommended in regular areas, in order to lighten the algorithms and obtain more robust results.

The aim of this work was to carry out a comprehensive assessment of the impact of the number of GCPs on the absolute geometric accuracy of photogrammetric products. The results obtained are in line with those presented in several previous works in literature: clear gaps show in the DG cases, noting the need of only 3 GCPs for a reasonable georeferencing. An efficient results may be obtained

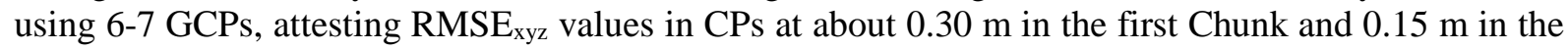
second one, which is practical for GIS applications. More than 15 GCPs are instead necessary to produce accurate cartographic works.

Further studies to make the photogrammetric process autonomously efficient will be carried out in the future, integrating the workflow with an automatic research of the optimal spatial and numerical distribution of GCPs.

\section{References}

1. Agüera-Vega, Francisco, Fernando Carvajal-Ramírez, and Patricio Martínez-Carricondo. 2017. 'Assessment of photogrammetric mapping accuracy based on variation ground control points number using unmanned aerial vehicle', Measurement, 98: 221-27.

2. Caroti, Gabriella, Andrea Piemonte, and Yari Pieracci. 2018. "Low-Altitude UAV-Borne Remote Sensing in Dunes Environment: Shoreline Monitoring and Coastal Resilience." In, 281-93. Cham: Springer International Publishing.

3. Gonçalves, J. A., and R. Henriques. 2015. 'UAV photogrammetry for topographic monitoring of coastal areas', ISPRS Journal of Photogrammetry and Remote Sensing, 104: 101-11.

4. Green, David R, Jason J Hagon, Cristina Gómez, and Billy J Gregory. 2019. 'Using Low-Cost UAVs for Environmental Monitoring, Mapping, and Modelling: Examples From the Coastal Zone.' in, Coastal Management (Elsevier).

5. Irvine, M, G Roberts, and L Oldham. 2018. 'Assessing the Applicability of Unmanned Aerial Vehicle (UAV) Data in Environmental Monitoring of Coastal Environments: St. David's, Newfoundland'.

6. James, M. R., S. Robson, S. d'Oleire-Oltmanns, and U. Niethammer. 2017. 'Optimising UAV topographic surveys processed with structure-from-motion: Ground control quality, quantity and bundle adjustment', Geomorphology, 280: 51-66.

7. Manfreda, Salvatore, Petr Dvorak, Jana Mullerova, Sorin Herban, Pietro Vuono, José Juan Arranz Justel, and Matthew Perks. 2019. 'Assessing the Accuracy of Digital Surface Models Derived from Optical Imagery Acquired with Unmanned Aerial Systems', Drones, 3: 15.

8. Manfreda, Salvatore, Matthew F. McCabe, Pauline E. Miller, Richard Lucas, Victor Pajuelo Madrigal, Giorgos Mallinis, Eyal Ben Dor, David Helman, Lyndon Estes, Giuseppe Ciraolo, Jana Müllerová, Flavia Tauro, M. Isabel De Lima, João L. M. P. De Lima, Antonino Maltese, Felix Frances, Kelly Caylor, Marko Kohv, Matthew Perks, Guiomar Ruiz-Pérez, Zhongbo Su, Giulia Vico, and Brigitta Toth. 2018. 'On the Use of Unmanned Aerial Systems for Environmental Monitoring', Remote Sensing, 10: 641. 
9. Mayer, Cedric, LM Gomes Pereira, and Thomas P Kersten. 2018. "A Comprehensive Workflow to Process UAV Images for the Efficient Production of Accurate Geo-information." In IX National Conference on Cartography and Geodesy.

10. Papakonstantinou, Apostolos, Michaela Doukari, and Konstantinos Topouzelis. 2017. Coastline Change Detection Using Unmanned Aerial Vehicles and Image Processing Techniques.

11. Papakonstantinou, Apostolos, Konstantinos Topouzelis, and Gerasimos Pavlogeorgatos. 2016. 'Coastline Zones Identification and 3D Coastal Mapping Using UAV Spatial Data', ISPRS International Journal of GeoInformation, 5: 75.

12. Rangel, José Manuel Galván, Gil Rito Gonçalves, and Juan Antonio Pérez. 2018. 'The impact of number and spatial distribution of GCPs on the positional accuracy of geospatial products derived from low-cost UASs', International Journal of Remote Sensing, 39: 7154-71.

13. Sanz-Ablanedo, Enoc, Jim H. Chandler, José Ramón Rodríguez-Pérez, and Celestino Ordóñez. 2018. 'Accuracy of Unmanned Aerial Vehicle (UAV) and SfM Photogrammetry Survey as a Function of the Number and Location of Ground Control Points Used', Remote Sensing, 10: 1606.

14. Saponaro, Mirko, Eufemia Tarantino, and Umberto Fratino. 2018. "Geometric Accuracy Evaluation of Geospatial Data Using Low-Cost Sensors on Small UAVs." In Computational Science and Its Applications ICCSA 2018, edited by Osvaldo Gervasi, Beniamino Murgante, Sanjay Misra, Elena Stankova, Carmelo M. Torre, Ana Maria A. C. Rocha, David Taniar, Bernady O. Apduhan, Eufemia Tarantino and Yeonseung Ryu, 364-74. Cham: Springer International Publishing.

15. Scaioni, M., J. Crippa, M. Corti, L. Barazzetti, D. Fugazza, R. Azzoni, M. Cernuschi, and G. A. Diolaiuti. 2018. 'Technical Aspects Related to the Application of SfM Photogrammetry in High Mountain', Int. Arch. Photogramm. Remote Sens. Spatial Inf. Sci., XLII-2: 1029-36.

16. Skarlatos, D., E. Procopiou, G. Stavrou, and M. Gregoriou. 2013. "Accuracy assessment of minimum control points for UAV photography and georeferencing." In First International Conference on Remote Sensing and Geoinformation of Environment, 9. SPIE.

17. Talavera Madrigal, Lara, Laura Del Río, Javier Benavente, Luis Barbero, and J. López-Ramírez. 2018. UAS as tools for rapid detection of storm-induced morphodynamic changes at Camposoto beach, SW Spain.

18. Tarantino, Eufemia. 2012. 'Monitoring spatial and temporal distribution of Sea Surface Temperature with TIR sensor data', Italian Journal of Remote Sensing/Rivista Italiana di Telerilevamento, 44.

19. Valentini, Nico, Alessandra Saponieri, and Leonardo Damiani. 2017. 'A new video monitoring system in support of Coastal Zone Management at Apulia Region, Italy', Ocean \& Coastal Management, 142: 122-35.

20. Valentini, Nico, Alessandra Saponieri, Matteo Gianluca Molfetta, and Leonardo Damiani. 2017. 'New algorithms for shoreline monitoring from coastal video systems', Earth Science Informatics, 10: 495-506.

Information about authors:

Mirko Saponaro. Master's Degree in Civil and Environmental Engineering. PhD student. Polytechnic University of Bari. Via Orabona n.4. 70125 Bari (Italy). +39 3490062790. mirko.saponaro@ poliba.it . Fields of interest: UAV imagery processing. Photogrammetric Accuracy.

Eufemia Tarantino. Associate Professor of Geomatics. Polytechnic University of Bari. Via Orabona n.4. 70125 Bari (Italy).+39 0805963417. eufemia.tarantino@poliba.it Fields of interest: Remote Sensng. GIS. Cartography. Alessandro Reina. Assistant Professor of Environmental Geology. Polytechnic University of Bari. Via Orabona n.4. 70125 Bari (Italy). +39 0805963493. alessandro.reina@poliba.it Fields of interest: Giacimentology, Mineral resources, Coastal erosion

Giuseppe Furfaro. Surveying Technician. external collaborator. g.furfaro@stf-survey.com . Fields of interest: UAV survey, Photogrammetry, GNSS survey.

Umberto Fratino. Full Professor in Hydraulic Enginering. Polytechnic University of Bari. Via Orabona n.4. 70125 Bari (Italy). +39 0805963321. umberto.fratino@poliba.it Fields of interest: Environmental modelling, water resources, hydrogeological risk. 